\title{
Problems in Combing Translation Practice and Guidance Theory in the Graduation Thesis of MT (Master of English Translation)
}

\author{
Zhang Yu \\ Xinyang Agriculture and Forestry University, Xinyang, Henan Province
}

Keywords: guidance theory; translation practice; neglect; combine

\begin{abstract}
This paper tries to analyze problems in combing translation practice and guidance theory in the Graduation Thesis of MT (Master of English Translation) students and give some suggestions to improve this situation, the author hopes that the importance of learning translation theories can be realizeds, not only the students, but also the professors and teachers, and then finds a good way to combine it with practice. Although the goal of MT subject pays more attention to practice, the study of translation theories cannot be neglected. The paper is composed of three parts, part one is an introduction to MT students training mode, in which relation between guidance theory and translation practice is explained; part two presents problems in completing their translation practice report, and here an example is given; in the last part, the author proposes some suggestions for improving the situation, hoping they can be helpful for scholars' reference.
\end{abstract}

Master of Translation and Interpretation (MTI) is an emerging subject which has quickened the process of cultivating professional talents in the field of translation. Being different from academic-oriented postgraduate student, it focuses more on the translation practice training rather than the theoretical study, thus lead to MTI students' relatively weak or even superficial understanding of relevant translation theories. To be more specific, we take MT students as an example, in doing their translation practice report, they may have doubts in choosing the guidance theory to support their translation, and still have a lot of problems in perfectly combing the theory and the practice. Factually, students have the awareness of eliminating the "two-skins" phenomenon, but how to realize it and make the thesis more rigorous is an important issue that calls us to pay great attention to. Here the author tries to analyze the issues and propose some suggestions to cope with it.

\section{A Brief Introduction to MT Students Training Mode}

The way MT cultivates talents is a picture of specialist education, which is a practice-oriented mode to breed professional translators. However, the prospects of the MT students are usually not the same as what they have ever imagined. In order to be capable to do translation work in any circumstances, MT students are required to recite a lot of materials, including a great many fields such as customs and traditions of a certain place, differences between Chinese and western cultures, statements and speeches given by the leader, current situation of economic growth, recent changes in global society etc. Facing such sophisticated materials, MT students must have great patience and convictions to read through and think carefully about the text, and then refer to some relevant data via various media, even turn to some others who are experienced and learned. The twists and turns in exploring the original text can be great barriers, but to understand the original text is the first step in translation. They are required try to grasp the essence of the sentence, paragraph and passage, and then convey it to the target text in their own words without loss in the meaning. Meanwhile, their translation should be as faithful as possible to the original text. However, professors and teachers, as well as Mt students themselves, have more or less ignored the significance of studying translation theories. Translation theory is a kind of comprehensive knowledge which has been concluded by philosophers, linguists, translators, it usually originated in the ancient times, perfected and developed by lots of scholars, so it is of great importance to understand the history of translation, laws, principles and also techniques in it. As what we have mentioned above, MT 
students just work continuously on translation practice and spare little or even no time on learning translation theories, they can hardly meet the requirements of "good translation". They are expected to meet the requirements of translation, which is even regarded as an art in the eyes of some scholars, but their foundation was not laid in a solid way, it is really contradictory.

Here the foundation for a high building refers to their understanding of translation theory. Whenever we mention theory, we argue that theory must be closely related to practice, or in other words, it is practice that makes theory meaningful. But have we ever wondered why we have seldom heard people emphasize that practice must be based on theory? It is an absolute truth and there is no need to doubt about it. Complicated work should be done after careful design and overall schedule, only after we have understand the ways and methods to deal with problems can we take actions, right?

In the campus, few MT students would like to read books in the classroom or the library, instead, they prefer to do some translation work as a volunteer in some activities such as meetings, exhibitions etc. They consider them good for improving their translation skills and can gain much experience, of course, their teachers also encourage them to do so. However, why not think more about the future? Translation calls knowledge input, when knowledge is not sufficient to be extracted when you need, what an embarrassing situation you will in! Inspiration comes from abundant knowledge reserves, for MT students, the knowledge reserves can be explained as all kinds of translation theories such as Interpretive Theory, Functional Equivalence Theory etc. By mastering them, MT students can be continuously enlightened, and their inspiration can hardly dry as theory is the origination of translation activity. If we stress it from a specific perspective, it is absolutely needed when doing translation practice report in their graduation thesis.

\section{Problems in the Translation Practice Report}

What has been stated above can be seen as a general introduction to the current situation of MT students. Here we will pay attention to specific problems on doing translation practice report which is usually selected as a form of MT student's graduation thesis. Translation practice report calls MT student to elaborate the details in completing the thesis and the translation techniques and skills adopted in it by giving examples, and the thesis usually contains the following parts: the depiction of the translation task, description of the translation process, introduction to the guidance theory, corresponding techniques and skills used in the translation and conclusions or the purposes of writing this thesis. For MT graduates, the most important thing is to clarify how you combined the guidance theory with your translation practice, or in other words, in which way you got the translation version with the guidance of a certain theory.

However, MT students have great difficulties in finishing a paper that can meet the requirements. For example, a student tries to write a paper named A Report on the Translation of Into Porcelain Pillow: 101 Tales from the Records of Taiping Era (Excerpts) under the Guidance of Interpretive Theory. Obviously, the author must analyze the style and features of the materials he chose, determine which theory is proper to guide the translation practice, consider carefully about the way of combining the theory and practice, then he should narrate the interesting stories and anecdotes and his translation version, after which the way he understands the original text and the translation methods and techniques which are used in the translation should be elaborated. Into Porcelain Pillow: 101 Tales from the Records of Taiping Era records the stories and anecdotes in the ancient times, so great efforts have to be made to achieving the equivalence in meaning between original text and target text. The students will inevitably meet difficulties in both understanding the text and conveying the meaning faithfully to the target readers. Facing such situation, students' attention will be first paid on understanding the original text, and then they will work hard to realize obtaining translation version that can successfully convey the meaning of the original text. However, it is an ancient Chinese book in which a lot of complicated words differ in meaning with modern Chinese, leading to the focus on exploring the meaning of every word and sentence, and then being faithful and expressive; as for elegance, they will pay less attention to it as the original text is hard to understand. 
The first point we should consider is how to choose a proper theory to guide the translation. Considering the features and style of the book, Interpretive Theory, Skopostheory, Functional Equivalence can be taken as primary options. Besides, arguments of scholars as George Steiner, Katharina Reiss, Hans and Vermeer, Christiana Nord must be studied carefully to decide which translation theory is finally chosen as the guidance theory because their statements should not be contradictory with the translation, or the theory will be the worm that lowers the quality of our thesis. After determining it, it can be a guideline in the process of translation. The second point is to give some examples that can represent your translation characteristics and style, and those examples must reflect the way you combine the theory with practice. In this book, some interesting things about animals and plants that are relatively different from our present recognition are recorded, legendaries and tales about fairy immortals, monsters and ghosts are also presented in front of the readers, and a great many stories of ancient places, kingdoms, minorities are shown to the readers, calling for detail interpretation to avoid misunderstanding and the mistakes brought about by linguistic ambiguity. Therefore, for the examples given in the thesis to support our arguments, we can adopt various translation techniques as literal translation with annotation, transliteration and free translation on the basis of being loyal to the guidance theory. To illustrate, about Dayi Guo [Arab Empire], we should add an annotation to interpret its geological location, the overall situation of it and where it belongs to at present. In short, all strategies and techniques we adopted and all procedures we do should conform to the guidance theory's leading function.

The author has elaborated the course of doing translation practice report and procedures in finishing it in an explicit way. The key point is to complete it according to the right steps, namely deciding the guidance theory goes first and then comes the translation; never consider which theory should be adopted to perfect the practice report after finished it. That's a behavior is to put the cart before the horse, an error that all MT students must attach great importance to.

Chinese, as the mother tongue, is the foundation for our language learners. Factually, when doing C-E translation, if we say English decides whether the expressions in translation conforms to the habit of expression in foreign culture, then Chinese determines whether you can thoroughly understand the original text in the right way, or in other words, the distinction between right and wrong. $\mathrm{Xu}$ Yuanchong once argued that translation conveys the beauty of Chinese, which is extremely critical for translators. It can be interpreted from two perspectives: it can be regarded as a standard whether a translator can meet requirements of good translation; and it also emphasizes the importance of studying Chinese classic works such as The Romance of the Three Kingdoms, The Encyclopedia of China, Complete Tang Prose etc. We should attach great importance to approach the linguistic beauty of our language which contains the profound cultures of Chinese nation. The materials cover a wide scope of biographies, institutions, laws, official names, customs and many other things, these calls a post-graduate student need to learn consciously. Someone will propose different opinions that target text is the standard to judge the translation work's quality. We cannot deny in doing translation, the target language is directly showed to the readers, but mastering Chinese greatly helps translator to grasp the essence of Chinese text, especially in some texts which are difficult to understand, just like the book we chose as an example in the last part. For example, the author once translated a prose named Don't Be too Harsh about Love, in which some hidden feelings and implications are contained in the writer's reflections about the things he had experienced, and that calls the translator to have a relatively good mastering of Chinese.

Some will propose such statements like your Chinese should be good when doing some C-E translation as literature work as prose, poems, novels etc, but when it comes to materials in our daily work, it doesn't call for professional knowledge and skills. Here I want to stress the subjects in the entrance examination for post-graduate study that MTI students have to pass, in which "Chinese Writing and Encyclopedic Knowledge” is clearly listed there, right? If Chinese doesn't work in most situations, why it emerges in the entrance examination? It apparently shows the importance of learning Chinese, it is a way to make you better understand the text and avoid some mistakes that you should not make. What's more, it is a foundation for realizing good combination of the theory and practice because understanding is the start of all. 
However, here is a misunderstanding that the author intends to emphasize by taking the opportunity of stating the importance of learning Chinese and its relationship with mastering English, which is similar with that between the theory and practice. That is to say, the relationship between the two is dialectical, and we need to pay attention to both instead of overemphasizing one or ignoring any of the two. Some may purely engage in the research of theories, but how theory comes without the experience obtained from practice? In general, theory for theory's sake is meaningless. Of course, there are a lot of people arguing that translation theories make no sense and there is no need for mastering them, what translators should do is to gain experience from continuously translation and form their own styles and characteristics. From the author's perspective, we should at least have a standard for judging whether the translation version conforms to the original text and the taste of target readers, so such a view is not valid because it runs counter to the public's perception and also the laws of recognition. Therefore, at the time of learning theories, we must balance it with the translation practice as they two work together in achieving the goal of successfully cultivating professionals in translation filed.

\section{Suggestions for Improving the Situation}

Since we have known that problems in combing guidance theory and translation practice are largely caused by ignorance and superficial understanding of translation theories, we should lead MT students to attach more importance to learning theories. We often say "Combing theory with practice", which apparently shows the importance of theory because it goes first and then comes practice.

It also works for MT students, when preparing their graduation thesis, they should firstly think about what text they choose as the original text, and then they should decide which translation theory is proper to guide the translation practice instead of just translating without considering the guidance theory. What the author wants to emphasize is never think about how to make the translation fit into a certain theory, it should be done conversely. That calls teachers to share their more information and understandings about general translation guidance theory; ask them to realize the thoughts and conclusions proposed by famous scholars and translators; give some work to rationally make use of theory to enlighten students in doing translation practice. As students, they should have the awareness of approaching the categories of translation theories and the development processes of them, which will help them better understand in what situation the certain theory can be applied and then they are clear about what specific translation techniques should be adopted. Group-work can be implemented, by presentation given by the representatives of each group, students can learn more about various theories and this makes work more effective. Of course, action is the most important thing for them, only by continuously practice, can they eliminate the obstacles in combing translation practice with guidance theories.

Competitions with the subject of translation theories and lectures are effective forms to stimulate students' activity to learn translation theories. In that form, students rely on their own exploration and give their opinions, the collective learning atmosphere drives them to study hard. Also, homework that asks students to translate the given text with a certain guidance theory is also helpful to cultivate their competence; with time goes by, they will contact more and more theories, so the knowledge structure is getting more and more complete and comprehensive, that makes them able to meet the requirements of a good translator, or at least be a qualified translator. Of course, there are also many different ways that can arouse teachers' and students' attention to enhancing the learning of translation theories and assign the thesis according to the right procedures, what the author gives in this article will be the base for further research, and the study on this subject needs to be expanded to a larger scope if we want to witness a brighter futures of MT students.

\section{References}

[1] Peng Qinglong, To Explore the Construction of the Quality Standard and Evaluation System for the Training of Graduate Students Majoring in MTI degree programs [J]. Chinese foreign language, 
2017, 14(03):74-81.

[2] Chen Lin, Zhang Yan, Study on the Writing Model of MTI Students' Graduation Thesis [J]. Chinese Translation, 2017, (06).

[3] Lv Liangqiu. Translation of Lantern-Riddles in a Dream in Red Mansions from the Perspective of Interpretive Theory [A]. Northeast Asia International Symposium on Language, Literature and Translation, Shenyang, China. Proceedings of the Fifth Northeast Asia International Symposium on Language, Literature and Translation(NAISOLLT)[C]. Northeast Asia International Symposium on Language, Literature and Translation, Shenyang, China: 2016:7.

[4] Liu Xiaofang. Brief Introduction to Chinese Translation Theories [J]. Overseas English, 2012(06):143-145.

[5] Li Xiaoling. The application of translation and translation techniques in English teaching. [A]. Information Engineering Research Institute, USA. Proceedings of 2012 2nd International Conference on Applied Social Science (ICASS 2012) Volume 4[C]. Information Engineering Research Institute, USA: 2012:4. 